\title{
Research on the Communication of Major Events and the Construction of Xiamen's City Image-—Take Twitter as an Example
}

\author{
Xueting $\mathrm{He}^{1, *}$
}

${ }^{1}$ Huaqiao University, Xiamen, China, 361010

*Corresponding author.Email:1835111007@stu.hqu.edu.cn

\begin{abstract}
The international city image depends more on the publicity and communication activities of multiple subjects. Xiamen has many resources for major events. How to transform resource advantage into image advantage and how to implement effective communication strategies have become a vital topic of Xiamen's international image communication. In this paper, through content analysis and literature research methods, the author takes Twitter accounts of major events, like China International Fair of Investment and Trade and Xiamen International Marathon, as the research focus, both of which are named as "CIFITinXiamen " and "@XiamenMarathon " respectively. Besides, the thesis points out the shortcomings and improvement direction for them. The thesis concludes that Xiamen should firmly grasp the opportunity of publicizing major events through overseas social media platforms. From the characteristic of the operation frequency and content, as well as the setting of topic labels, they all can enhance follower stickiness and engagement if being used effectively. Traffic is the focus for social media when publicizing major events in Xiamen abroad. Moreover, it has enormous potential to step further from improving its international image.
\end{abstract}

Keywords: major events, city images, international communication, Twitter accounts

\section{INTRODUCTION}

The 14th Five Year Plan set goals for Xiamen's development and it is committed to promoting the construction of the international shipping, trade, and Tourism Exhibition Center. At the same time, international reputation and recognition have become indispensable for Xiamen to build an international modern city. Xiamen is located on the southeast coast. As the first batch of special economic zones to open up, it always has good international resources. However, only mastering the resource advantages is far from enough. Its international reputation is inseparable from the international communication of the city's image. The city image is composed of the objective reflection of the city and people's subjective cognition. From the perspective of international communication, the boundary between geography and culture will block people's direct objective experience of the city. The international city image depends more on the publicity and communication of activities of multiple subjects.
At present, overseas social media accounts for the major events in Xiamen have a slight impact on the international communication of Xiamen city's image radically due to the deficiency of system management. This situation not only fails to give full play to the role of overseas social media platforms, but also is not conducive to transforming Xiamen's "resource advantages" into "international image advantages". This thesis aims to fill the gap in this research field in Xiamen, analyze the international communication of overseas social media accounts of major events in Xiamen, and then give suggestions to make up for this "weakness". This thesis will also take the overseas new media account of major events in Xiamen as the research object. Content analysis and literature research methods are research methods. Firstly, the content analysis method is used to explore the correlation and possible causality between international communication of urban image and major events. Then, it made a comparison among three specific research objects the official Twitter accounts of Xiamen fair, Xiamen marathon, and Xiamen culture and Tourism 
Bureau. Meanwhile, the concepts which are defined as "Media false events" "major events" "media Reengineering" "Collective memory" in the thesis are sorted out by the literature research method. Combined with Durkheim's concept of social psychology "collective memory" and Katz's book "Media Events"[3], the author believes that the media process of major events refers to artistic processing and creation, and mutual relationship with cultural capital and political system in society. However, it is not a false event without real occurrence, but a real event with symbolic significance. It is a self prophecy that attracts the attention of the media and society. Media reengineering attracts the attention of the outside world, making audiences quickly form the impression of major events, and virtually affects the audience's construction and acceptance of the city image.

\section{OVERVIEW OF CITY IMAGE AND MEDIA}

In this part, the paper will discuss the city image and media in details, including city image and international communication and major events.

\subsection{City Image and International Communication}

The city image is composed of the objective reflection of the city and people's subjective cognition. From the perspective of subject-object dualism, city image has the characteristics of fluidization and relativity, which means that city image is a dynamic cognition and has different construction of different cognitive objects. The city image depends more on people's awareness and recognition tendency of the city. The city image is born with the city but not completely dominated by the city.

From the perspective of traditional international communication, geographical boundaries and cultural barriers block people at home and abroad to improve their cognition of a certain city. The city image mainly depends on the construction of traditional media discourse. The emergence of online social media platforms not only breaks through the limitation of distance in the geographical sense, but also provides the possibility for smooth cognition in the cultural sense. In addition, the incoming technology of new media also breaks the discourse monopoly of traditional media. Users can participate in and influence the construction of urban images through spontaneous communication activities, such as forwarding, likes, comments, and posts.

\subsection{City image and major events}

Major events refer to activities sponsored or authorized by the government and covering many fields relying on certain resources and strengths of the government[1]. They can affect the trend of urban development and leave broad and profound social repercussions. At the same time, major events have the characteristics of high social participation, and are easy to attract international media reports and large investments. These characteristics are also an aspect of city's image.

Major events in Xiamen are an important part of the construction of Xiamen city image. "CIFIT" in the political and economic field and the "Xiamen International Marathon" in the field of sports activities have become resource advantages for the dissemination of Xiamen's urban image. The scholar named $\mathrm{He}$ Guoping puts forward that the city image should be based on the city's positioning and constructed through appropriate media and cultural strategies[2]. Therefore, how to transform "resource advantage" into "image advantage" and implement effective communication strategies has become an important topic of Xiamen international image communication.

\section{OPERATIONAL ANALYSIS OF THE TWITTER ACCOUNT - "CIFITXIAMEN" AND "XIAMEN MARATHON"}

At present, overseas social media is one of the main means of international dissemination of national city image, with Facebook, Twitter, and Youtube as the main communication platforms. Overseas social media has a wide audience. In this part, the CIFITXiamen and Xiamen marathon will be analyzed in details.

\subsection{The Role of Overseas Social Media in the Dissemination of Xiamen City Image}

According to the 2020 global digital report, the number of global social network users will reach 3.8 billion by 2020. Among them, Facebook, for example, has 2.9 billion monthly active users, accounting for about one-third of the global population. By 2021, a total of 51 cities in China have opened 293 accounts on overseas social platforms such as Facebook and Twitter, and released more than 3 million website information. Among them, Xiamen culture and tourism bureau registered the most successful social media account and it has gained a huge fan group for marketing overseas. The contents of the account select one aspect of the city image of Xiamen, like the local customs and folk culture of Xiamen. Besides, it uses netizens' common pursuit of beauty, nature, and food to weaken the tendency of politics and ideology. As a result, it achieved the trick of marketing. The average praise of each tweet is more than 100. Meanwhile, the Twitter account named "visit Xiamen" ranks first among 80 official accounts for 40 overseas marketing cities in China. Although this 
account is a successful case of spreading Xiamen's city image, it is only an angle of Xiamen's image after all. When it comes to Xiamen's major events, such as the "CITIF fair", "marathon" and "BRICs meeting", Xiamen made strategic mistakes in strengthening the connection between major events and the city's overseas marketing through overseas social media platforms. That is why Xiamen has difficulties in transforming "resource advantage" into overseas "image advantage", and almost no one cares about the account specially set for major events.

\subsection{Operation Analysis of Twitter Account @CIFITinXiamen of Xiamen International Investment and Trade Fair}

The official Twitter account@CIFITinXiamen of Xiamen International Investment and Trade Fair entered the platform in August 2019. Its operation contents, shortages and suggestions are presented as follows.

\subsubsection{Overview of operation contents and shortages of @CIFITinXiamen}

By July 2021, there were more than 3200 fans. Since accounts are registered, $80 \%$ of the tweets have not been forwarded or commented on, and the stickiness of fans is weak. During the period of the Xiamen International Investment and Trade Fair, the account will release the contents about exhibition planning, exhibition arrangement, and exhibition news of the fair. During the period without fairs, the tweets released by the account were mainly original, which introduced the recent exhibitions in Xiamen and some data news in the economic field. Most of the reprinted content came from the interpretation of economic and trade policies by China Daily[6].

The disadvantages of the account are obvious. It is observed that the frequency of tweeting on its account is not on a fixed frequency. The frequency of tweeting on different days can differ by up to seven times. At the same time, the account takes the twitter maker as the center, and does not pay attention to its media role orientation for international audiences. When it comes to oversea market, it is impossible to simply transplant domestic content completely to overseas media. Although social media is widely used overseas, the account content is still based on hard economic data and policy interpretations, and these contents will not achieve good communication effects. Account managers ignore Twitter as a social media, which has its own unique rules of dissemination.

\subsubsection{Operation Suggestions for @ CIFITinXiamen}

The author believes that the hard news of the account should be softened and the content maker should adjust the narrative mode from the perspective of the audience. The content maker should emphasize the point that can catch the eyesight of the audience. For example, it is an effective way to timely collect and sort out the copy-writing feedback that has to do with the CIFIT to adjust the communication content, as well as setting up an "online round table" for CIFIT related topics to encourage interaction with the audience. In addition, the content maker should release more CIFIT videos and use a variety of TV and stunt to beautify the videos visually and symbolically and give overseas people a more perceptual feeling of visiting the venue. Video is also discourse rhetoric and direct media expression to reduce cultural differences so that the content is convenient for overseas people to understand and cognitive.

The operation of the @CITIFinXiamen account during CIFIT focused on publicizing the Xiamen fair itself, without integration of city image and major events. The city's cultural and environmental publicity can help to build a good city image and attract overseas people's interest in Xiamen International Investment and Trade Fair. Xiamen is a Chinese special economic zone approved by the State Council and an important central city and port along China's southeast coast. Xiamen International Investment and Trade Fair have inherited the spirit of Xiamen's opening-up and have become a landmark for international trade exhibition and Exchange Fair since the reform and opening up. What is more, the beautification and publicity of Xiamen's urban style are also a powerful means to build a green and livable city image of Xiamen. General Secretary Xi once praised Xiamen as "an ecological garden city with high beauty". Xiamen ranks first in terms of landscape and ecological civilization index in China, and enjoys international reputations such as "International Garden City" and "UN-Habitat Award". During Xiamen International Fair, the international media gathering should seize the exposure effect and harness the social media to build Xiamen's city image into a tourist city yearned for by overseas tourists.

\subsection{Operation Analysis of Twitter Account @ XiamenMarathon of International Xiamen Marathon Competition}

Xiamen marathon's official Twitter account @ XiamenMarathon entered the platform in December 2015. The figure for fans likes and forward is similar to @CIFITinXiamen.

\subsubsection{Overview of Operation Contents and Shortages of @XiamenMarathon}

The author observed the content of the account in the past two years and found that the content of the account 
is designed and organized from the perspective of humanities, environment, and sports, which is unique in many major event operations. The tweets are lively, with an exclamatory tone and colloquial expression. In the retouching of the content, it can be seen that the makers paid great attention to the spread of emotion, and "happy" and "happiness" became high-frequency words of account twitter content[7].

The account published many runner stories, such as photos of runners kissing and pictures of family and friends hugging each other. The runner story jumps out of the reporting framework of traditional sports events, paying attention to and showing the runner's real life. The runner is no longer a rigid running symbol. In the operation and development of the event, the runner is an individual with dignity and independence and enjoys the emotional companionship of love and family affection. Attaching importance to the ethical values of friendship, love and traditional family makes the publicity of the event more humanistic.

Low attention shows that there are still large operational errors in the account. First of all, although Twitter presents an expression of "positive energy" and exudes a humanistic atmosphere, most tweets show a homogenization trend, which is warm but lacking in depth. Secondly, the topic tag independently created by the account lacks the influx of the first batch of traffic and does not follow the hot topics for publicity, which is not eye-catching. For instance, during the epidemic period when marathon competition is held, the operation of accounts is active online, but it would be silent when there are no events. It is not conducive to the accumulation of long-term fans.

\subsubsection{Operation Suggestions for @Xiamenmarathon}

Given the problems that concerned about the homogenization trend and lack of depth on the account, the author believes that the "marathon" itself focuses on "Sports". To keep the content vertical in the "Sports" field, it is necessary to excavate a continuous stream of materials, which requires the operation team on the field to collect a large amount of information. After having the materials, it becomes particularly important to dig deeply or bring forth new ideas. For example, the activities of "leaving only the beauty without garbage" and "green running" show value guidance in their tweets, but do not highlight the relationship with the positioning of Xiamen as a "green and livable" city. As we all know, Xiamen is the layout site of the marathon track and the foundation of the "marathon". However, if only the simple connection of "marathon green" is scattered in a few tweets, readers will feel that there is a lack of information that can be extracted from the tweets. At the same time, the author gives suggestions on "setting topic labels". Although "ride a wave" is a derogatory term in the so-called "fan culture", it's effective when operators use it with a correct attitude. That's is a good way to solve problems of just getting few fans. For instance, to improve the attention of accounts by riding a wave, the operators can label topics according to the current hot spots, or forward with the help of an account with a high number of fans. Finally, only by doing a good job in the long-term update operation of the account can we enhance the stickiness and activity of fans.

\section{COMMUNICATION STRATEGY OF CONSTRUCTING CITY IMAGE FOR MAJOR EVENTS}

Major events have a wide and profound impact on the city image because of their highly social participation, concentrated media coverage, and easy absorption of a large number of social resources. Major events will be externalized into the city image which will also affect the evolution and development of major events.

\subsection{Media Reengineering of Major Events}

Media events are neither false events nor news events. Between absolute truth and absolute falsehood, media events are among them. The media reconstruction of major events is to explore the development texture of major events and pick the most dramatic and important part to process the artistic transformation. The acceleration of media events not only deepens the audience's cognition and understanding of the original events, but also invisibly affects the audience's construction and shaping of the city image.

The process of externalization is not only the process of art and symbolization, but also a nonlinear communication process which is the media event discussed by the scholar Katz, who defines media events as a process that makes major events noble, profound, and cruel significance[3].

\subsection{Collective Memory of Major Events}

Collective memory is the sociological interpretation of individual memory. Major events are often accompanied by the emergence and liquefaction flow of collective memory. Some scholars believe that major events and places of occurrence together constitute the collective memory of the city[4]. On the social media platform, overseas users are in a state of absence, and the so-called collective memory prototype can only come from media events. Some scholars pointed out that users use social media to meet the needs toward the content and relationship[5]. From the perspective of communication, the production mode of communication content aimed at the needs of the audience is the best. In 
other words, the focus of media reengineering should be on the consideration of content and users' social relations. If the contents of communication are of much value and conform to the users' aesthetic taste, users will have spontaneous communication behavior out of social needs. Spontaneous communication behavior generates, strengthens and changes self-identity. When the self-identity is integrated through social media, it will produce collective identity, and then produce collective memory of media events. The collective memory of users is strengthened again and again in the virtual space, and the construction of the urban image is slowly completed. However, the formation of urban images is still a continuing process. Once the user's later experience is inconsistent with the original cognition, the urban image will be corrected.

\subsection{The Relationship Between the Media and Major Events}

Major events can often leave a wide and profound response to cities because of their high social participation, concentrated media coverage, and easy absorption of a large number of social resources. Elihugh Katz believes that what the public pays attention to and discusses is not a real major event, but a media fake event with noble, profound, and cruel significance[3]. Media false events are not false events without real occurrence, but real events with symbolic significance. They are self prophecies that attract the attention of the media and society.

Media is not a mirror reflection of social reality. The process of media is a process of artistic processing and creation, and a process of mutual relationship with cultural capital and political system in society. Media events come from reality, but they are not completely consistent with reality. The media reconstruction of major events is to explore the development texture of major events and pick the most dramatic and important part to process the artistic transformation. The reconstruction process of media from major events to media events will significantly affect the audience's cognition and understanding of the relationship between major events in the city and the city itself. The existence and strength of the correlation between them will be further solidified into the city image by the audience. Media reengineering attracts the attention of the outside world, makes the audience quickly form and deepen the impression of major events, and virtually affects the audience's construction and acceptance of the city image.

\section{CONCLUSION}

To sum up, in major events, there is still a lot of room to improve the construction of Xiamen city image on overseas social media platforms. Whether from the operation frequency and content to the setting of topic labels and improvement of attention and fan stickiness, they are the focus of the social media when publicizing major events in Xiamen abroad. The research aims to enlighten the Xiamen government media practitioners to deepen the audience's cognition and understanding of the major events through two feasible communication paths - - media reengineering and the creation of collective memory. At the same time, it can invisibly affect the audience's construction and the shaping of the city image. By focusing on the value and art for compliance with users need, it will be much easier for governments to contribute to the urban image through the use of mass communication. However, due to the author's limited ability, it is difficult to make a specific and detailed quantitative analysis on the description and research of the city image. Therefore, this paper will only conduct simple research on the city image of Xiamen from the perspective of qualitative analysis and text, which is the limitation of this paper. However, the author will carry out quantitative research later, for example, collecting the overall perception of domestic people and foreigners by issuing questionnaires about overseas social media accounts of major events in Xiamen, so as to put forward more comprehensive suggestions.

\section{REFERENCES}

[1] Kurui. Summary of research on the impact of major events on urban development [J]. Journal of Xi'an University of Arts and Sciences (SOCIAL SCIENCE EDITION), 2012,15 (05): 56-58

[2] He Guoping. Urban image communication: Framework and strategy [J]. Modern communication (Journal of Communication University of China), 2010 (08): 13-17

[3] Katz, Elihu. Media Events :The Sense of Occasion $1[\mathrm{~J}]$ Studies in Visual Communication, 1980, 6(3) $: 85,88$

[4] Tang Keqing, pan Jiali. City: major events and event space [J]. Times architecture, 2008 (04): 6-10

[5] Peng LAN: introduction to network communication, China Renmin University Press, 2017

[6] Twitter account "CIFITinXiamen" https://mobile.twitter.com/CIFITinXiamen

[7] Twitter account "CIFITinXiamen" https://mobile.twitter.com/XiamenMarathon 